\title{
Redes personales de aprendizaje (RPA) y competencia digital de estudiantes en educación obligatoria
}

\author{
Personal learning network (PLN) and digital competence among \\ compulsory education students
}

\author{
Sandra Dorado Gómez \\ Universidad de Santiago de Compostela \\ sandra.dorado@usc.es \\ Adriana Gewerc Barujel \\ Universidad de Santiago de Compostela \\ adriana.gewerc@usc.es
}

Recibido: 31/10/2016

Aceptado: $23 / 12 / 2016$

Publicado: 29/12/2016

\section{RESUMEN}

El presente artículo tiene como objetivo estudiar el estado del arte acerca de la influencia de las Redes Personales de Aprendizaje en el desarrollo de la competencia digital de los/as niños/as y jóvenes de educación obligatoria -entre 12 y 16 años-. Las Tecnologías de la Información y Comunicación (TIC) han adquirido gran relevancia en el contexto de la educación contemporánea provocando modificaciones en todos los aspectos del proceso educativo de los sujetos. Es por ello que la competencia digital se convierte en un elemento primordial de cara a emplear de manera crítica y segura las TIC en los distintos ámbitos de la vida. Una de las maneras de desarrollar dicha competencia es por medio de las Redes Personales de Aprendizaje o Personal Learning Network (PLN).

Para llevar a cabo dicho estudio se ha realizado una investigación documental buscando en las principales bases de datos educativas y analizando el contenido de aquellos documentos de relevancia mediante el software de análisis cualitativo Atlas.ti. Los resultados obtenidos proyectan un campo de estudio poco desarrollado. La faceta educativa de las Redes Personales de Aprendizaje en general, y su influencia en la competencia digital en particular, está escasamente abordada por las investigaciones. Existen algunas experiencias documentadas de este ámbito, aunque el número de las mismas es limitado.

\section{PALABRAS CLAVE}

Redes personales de aprendizaje; competencia digital; educación obligatoria; sociedad del conocimiento; estudio teórico-documental.

\section{ABSTRACT}

The article presented below, intends to initiate a study on the state of the art about the influence that Personal Learning Network have on the development of digital competence among compulsory education students -between 12 and 16 years old-. Information and Communication Technologies (ICT) have acquired great relevance in the context of the current education causing changes in all aspects of the 
subject's educational process. In this context, Digital Competence (DC) becomes a key element to face a critical and safe use of ICT in different areas of life. Personal Learning Network (PLN) is one of the ways to develop the DC.

To carry out this documentary research has been conducted on the main bases of educational data, analyzing by qualitative analysis software Atlas.ti. The results show us that it's a small developed field. The educational aspect of the Personal Learning Network in general and its influence on digital competence in particular, is barely addressed by the research. There are some documented experiences of this area, although the number of them is restricted.

\section{KEYWORDS}

Personal learning network; digital competence; compulsory education; knowledge society; theoretical- documental study.

\section{CITA RECOMENDADA}

Dorado, S. y Gewerc, A. (2016). Redes personales de aprendizaje (RPA) y competencia digital de estudiantes en educación obligatoria. RIITE. Revista Interuniversitaria de Investigación en Tecnología Educativa, 1, 26-38. Doi: http://dx.doi.org/10.6018/riite/2016/273231

\section{Principales aportaciones del artículo y futuras líneas de investigación:}

- Poner en valor la función de las Redes Personales de Aprendizaje como elementos clave en el desarrollo de la competencia digital.

- Resaltar la función del aprendizaje informal en la vida de los menores, siendo las redes de aprendizaje un aspecto a tener en cuenta en su proceso educativo.

- Recopilar y analizar diversas experiencias sobre esta línea de investigación, con el fin de dar visibilidad y difundir su relevancia en el contexto educativo.

\section{INTRODUCCIÓN}

El presente artículo tiene como objetivo realizar una revisión bibliográfica sobre el estado del arte de la influencia de las redes personales de aprendizaje en el desarrollo de la competencia digital en los/as niños/as y jóvenes de entre 12 y 16 años.

La importancia de este estudio radica en el incremento del interés en los contextos educativos informales, producido en los últimos años. Cuestión que evidencian autores/as como Coffield (2000) o Sangrà y Wheeler (2013) quienes destacan la relevancia del aprendizaje informal a la hora de crear entornos educativos que satisfagan las necesidades de los sujetos aprendices, en respuesta y adaptación a las nuevas condiciones establecidas por la sociedad. También Cuadros Muñoz (2015) otorga relevancia a aquel "aprendizaje que se desarrolla más allá del espacio cerrado del centro educativo y que apuesta por crear entornos y redes personales de aprendizaje (PLE y PLN, respectivamente)" (p.2). Dichos espacios emergen, según Castañeda y Adell (2013), a raíz de las necesidades e intereses formativos de los sujetos, asegurando una metodología individualizada. Aspectos como la motivación, interés o participación podrán incrementarse debido a que el sujeto adquiere un rol activo y capacidad de decisión en su propio proceso educativo.

Las redes personales de aprendizaje -tanto virtuales como presenciales- representarán el entorno social del colectivo destinatario, puesto que éstas "son un conjunto de conexiones entre personas y recursos -online y offline- que enriquecen nuestro aprendizaje" (Richardson y Mancabelli, 2011, p.2) o en palabras de Couros (2010) "la suma de todo el capital social y las 
conexiones que dan como resultado el desarrollo y la facilitación de un entorno personal de aprendizaje" (p.25). Es de relevancia por lo tanto, indagar en dichos espacios y sus características, con el objetivo de determinar la influencia real de los mismos en la competencia digital del colectivo destinatario.

Por otra parte, es necesario resaltar la importancia concedida a la competencia digital en nuestros días. Ésta, además de desarrollar la capacidad de aprovechar la riqueza de las nuevas posibilidades asociadas a las tecnologías, permite participar de forma significativa en la sociedad del siglo XXI. La OCDE (2014) prevé que las tecnologías seguirán siendo un motor clave en la creación de empleo, y asume el desarrollo de competencias en TIC como la más importante estrategia para las políticas de recuperación económica. Ya desde el año 2000, esta organización destacó la importancia de ciertas competencias basadas en el modelo de desarrollo soportado por Internet. En un primer momento, se focalizó la atención en las infraestructuras y el acceso de las personas a las TIC, esto es, la "posibilidad/dificultad de disponer de dispositivos conectados a Internet" (Camacho, 2005, p.63). Posteriormente, se genera una preocupación por el desarrollo de las capacidades y habilidades requeridas para la utilización de las TIC. Es importante por lo tanto, indagar en este último aspecto, con el fin de delimitar el nivel de competencia digital del colectivo destinatario de la investigación.

Además, el desarrollo de la competencia digital va más allá de un escenario o de una herramienta en concreto, sobre todo por las oportunidades ofrecidas por Internet. En esta línea adquieren relevancia, como citábamos anteriormente, los PLE y PLN consolidados como espacios heterogéneos, plurales y dinámicos. La conexión establecida entre éstos, con los distintos cambios producidos en la sociedad, los convierten en una gran posibilidad de aprendizaje informal para asegurar el desarrollo de la competencia digital de toda la población -independientemente de su edad-, así como una actitud de motivación y participación hacia el aprendizaje permanente.

Con esta base, el propósito de este trabajo es realizar una investigación documental que permita una aproximación al conocimiento desarrollado por las diferentes investigaciones sobre esta temática. Para ello, en las próximas secciones se abordará la metodología de trabajo, los principales hallazgos, sintetizando finalmente, las conclusiones.

\section{MÉTODO}

Para el estudio del estado del arte acerca de la influencia que las RPA tienen en la competencia digital de los/as niños/as, se ha adoptado la modalidad de revisión cualitativa sistemática (Green, Johnson y Adams, 2006), utilizando un riguroso proceso que identifica, evalúa y sintetiza estudios que posibiliten contestar las preguntas específicas y extraer, conclusiones sobre los datos recopilados (Melnyk, 2005).

Este proceso se ha articulado alrededor de las tres etapas establecidas en Galván (2006) a la hora de iniciar una revisión de la literatura, a saber:

Buscando la literatura: este proceso se centra en la selección de la bibliografía que servirá de apoyo para la presente investigación. Tendrá carácter selectivo, esto es, atendiendo a los resultados de la búsqueda anterior y a las características de los documentos (fecha de publicación, grado de relación con la temática, autor/es de relevancia en el ámbito de estudio, etc.). Se realizaron búsquedas en bases de datos electrónicas como: ERIC, Teacher Reference Center, Dialnet, Redalyc, JSTOR o Google Académico.

Revisando la literatura: esta fase se ha centrado en la revisión de la bibliografía recompilada hasta el momento y en su organización alrededor de las temáticas de 
investigación localizadas. Se han valorado un total de 180 documentos (ver Figura 1): artículos de revista, libros y capítulos de libro, tesis doctorales, actas de congresos, etc. Se han localizado dos temas centrales en estos recursos: uno de ellos centrado en las redes personales de aprendizaje, su conceptualización e implicaciones en el ámbito educativo; y el otro, focalizado en la función de estas redes como desarrolladoras de la competencia digital.

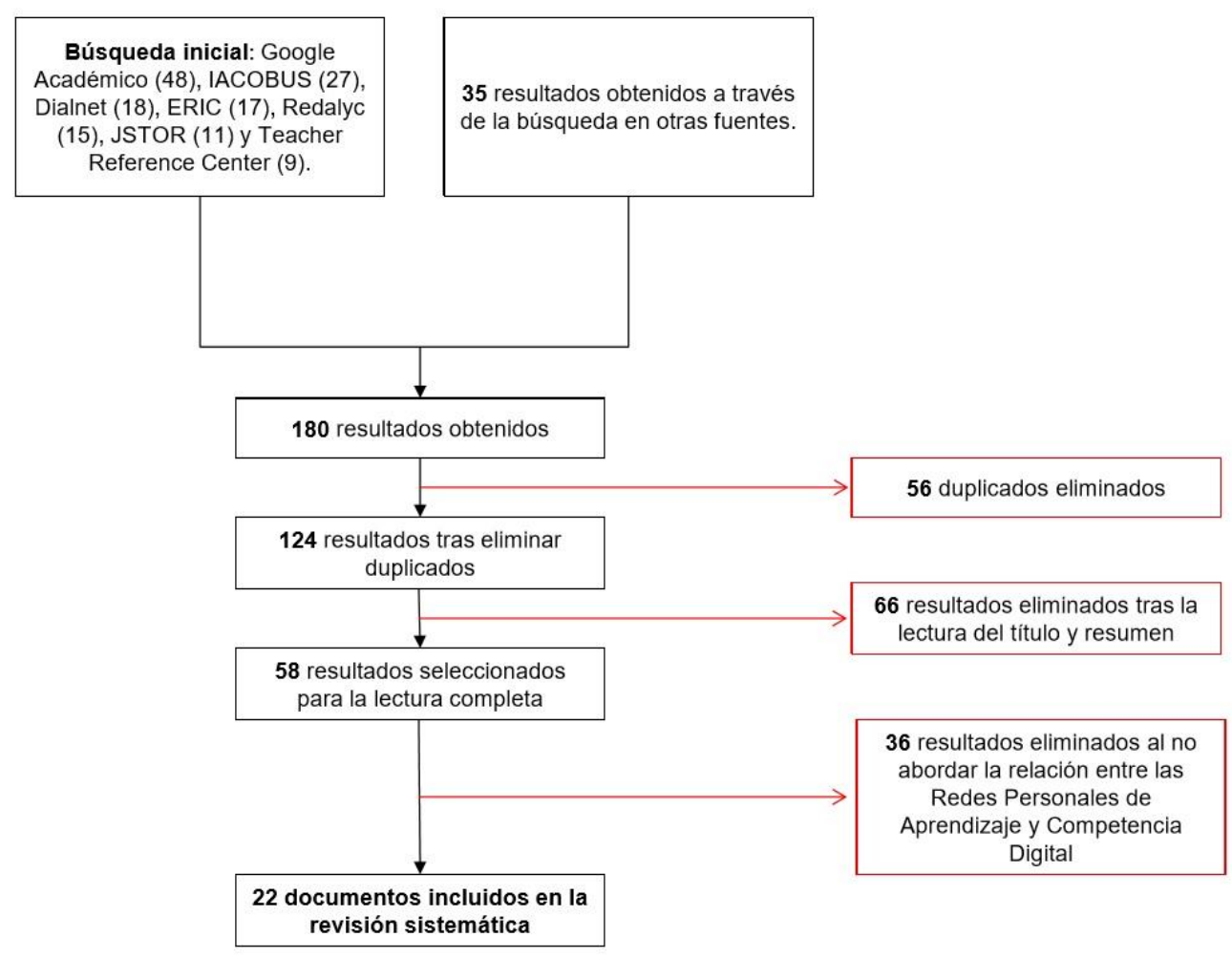

Figura 1. Diagrama de flujo de la información de las diferentes fases de la revisión sistemática.

Escribiendo la literatura: en esta última etapa se han revisado de nuevo los documentos recompilados en el gestor bibliográfico Zotero, con el fin de elaborar un informe analítico detallado del estado de los mismos. Para ello, se han teniendo en cuenta las fortalezas y debilidades de los estudios, sus hallazgos clave, así como las principales conclusiones extraídas a raíz del análisis. Con el fin de analizar la información contenida en los diferentes archivos, se ha optado por desarrollar el "método cualitativo de análisis de contenido" (Ruíz Olabuénaga, 1999) por medio del software para análisis de datos cualitativos Atlas.ti. Dicho análisis se ha llevado a cabo por medio de dos subetapas:

1. Reducción de la información (categorización y codificación): la reducción de la información se ha realizado a través de la búsqueda de temáticas comunes en los distintos documentos recopilados. Las operaciones llevadas a cabo para ello han sido la categorización - división y simplificación del contenido a unidades o categorías de acuerdo a determinados criterios temáticos - y la codificación - agrupación de la información obtenida en categorías que concentran las ideas, conceptos o temas similares descubiertos -.

2. Integración de la información: este procedimiento implica "relacionar las categorías obtenidas en la etapa anterior entre sí y con los fundamentos teóricos de la investigación" (Fernández Núñez, 2006, p.4).

\section{RESULTADOS}

La bibliografía sobre redes de aprendizaje en los sujetos de educación obligatoria es muy reducida, haciéndose más evidente cuando se atiende a su influencia en la competencia digital. 
Quizás esto es debido a que no se perciben como sujetos activos y participativos en estas redes, puesto que supeditan su acción a la supervisión y control de los adultos, principalmente de las familias y los docentes.

El conjunto bibliográfico recogido y analizado se organiza alrededor de dos temáticas: las investigaciones centradas en la influencia educativa de las RPA (18 documentos) y aquellas experiencias focalizadas en la influencia de estas redes en la competencia digital (4 documentos). Ambas se caracterizan por la heterogeneidad en el formato: artículos de revista, libros o capítulos de libros, tesis doctorales, presentaciones en conferencia, etc.

En cuanto al idioma de redacción de los documentos, las experiencias se encuentran escritas en castellano -todas ellas tienen lugar en España-; mientras que los documentos centrados en las RPA como estrategia educativa aparecen escritos en inglés, indicando una mayor presencia en países anglosajones (Estados Unidos, Inglaterra, etc.).

\subsection{Las Redes Personales de Aprendizaje (RPA) entre 12 y 16 años}

La mayor parte de la bibliografía consultada aborda la importancia que tienen las RPA en el entorno educativo. Es el caso de Rajagopal, Joosten-ten Brinke, Van Bruggen y Sloep (2011) quienes resaltan los beneficios que experimenta un sujeto inmerso en un PLN: contactos heterogéneos, disposición continua de recursos, participación en entornos colaborativos, etc. Casquero (2013) también considera la importancia de estos entornos a la hora de garantizar que los sujetos aprendan a buscar y dar respuesta a sus necesidades educativas fuera de la institución, considerándola esencial durante su desarrollo profesional.

Con respecto a las RPA, la mayor parte de los documentos abordan la importancia de estas en el contexto formal de la educación, más concretamente en lo que se refiere al entorno universitario (Salinas, 2004; Cabero, Barroso y Llorente, 2010). La intención de este artículo es "romper" con esta formalidad, indagando en las consecuencias en el aprendizaje informal. Se entiende que "el aprendizaje es social", puesto que la mayor parte de lo que aprendemos es gracias a aquellas personas próximas y no tan próximas a nuestro contexto diario. Jay Cross (2006) afirma que "el aprendizaje formal -clases, talleres y eventos en línea- es la fuente de solo el $10 \%$ y el $20 \%$ de lo que aprendemos" (p.2). Se hace fundamental por lo tanto, profundizar en el $80 \%$ restante en relación a los contextos educativos informales.

En estos contextos el grupo de iguales o pares adquiere una función de relevancia, puesto que los sujetos trabajan de manera colaborativa para alcanzar un determinado objetivo, responsabilizándose y comprometiéndose con su propio trabajo y con el de los demás. Autores como Apodaca (2006) y Domingo (2008) destacan que, a través del trabajo entre iguales, se desarrollan ciertas habilidades interpersonales como la negociación, el consenso, la argumentación estructurada y lógica, así como la capacidad para comprender los puntos de vista de los demás; mientras que otros como Cuseo (1996) consideran que aquellas personas que trabajan juntas tienden a implicarse más activamente en el proceso de aprendizaje.

Según Domingo (2008), la potencialidad de este tipo de aprendizaje frente al tradicional entendiendo tradicional como la posición de supremacía del adulto (profesor/a o familia)- se debe a que los iguales o pares están más cerca entre sí en lo que respecta al desarrollo cognitivo. De este modo, sus acciones y experiencias ante una determinada temática se igualan con las de sus compañeros/as. La importancia de este proceso es que no solo aprende la persona que se beneficia del aprendizaje de un igual, sino que este último refuerza su proceso educativo, puesto que adquiere un mayor nivel de comprensión. 
Este aprendizaje colaborativo en la red supone generar nuevas formas de participación que conforman aprendizaje informal al margen de las instituciones educativas. En palabras de Freire (2009), "los jóvenes adquieren, principalmente, sus competencias digitales y habilidades tecnológicas en estos espacios digitales informales y su actividad es eminentemente social y apasionada" (p.4). Indica también que los menores prefieren estos espacios puesto que les proporcionan autonomía y libertad en sus acciones y decisiones, y porque el estatus y la autoridad se determinan por sus habilidades y no por una jerarquía establecida.

Una investigación relevante en este campo, centrada en la participación de los jóvenes en los entornos digitales, es la denominada Living and Learning with New Media: Summary of Findings from the Digital Youth Project (Ito et al, 2008). Basada en un estudio etnográfico de tres años con sujetos jóvenes de entre 13 y 18 años tuvo el objetivo de "capturar las culturas juveniles y las prácticas relacionadas con los nuevos medios de comunicación, así como el contexto que les rodea: relaciones entre pares, dinámica familiar, etc." (Ito et al, 2008, p. 7).

Los resultados les llevaron a clasificar y organizar a los jóvenes en función de los "géneros de participación", esto es, los diferentes modos y convenciones a la hora de usar las Tecnologías de la Información y Comunicación. Establecen tres modos de participación: hanging out (pasando el rato con la tecnología), messing around (jugando un poco) y geeking out (utilizando con mucha frecuencia la tecnología).

El primero de estos géneros, hanging out, se caracteriza por la realización de actividades diversas en presencia de los iguales o pares. La mayor parte de éstas se encuentran relacionadas con los videojuegos online y con aquellas tecnologías de almacenamiento y compartición de información: redes sociales, plataformas de vídeos y música, etc. El objetivo de estos sujetos se focaliza en el establecimiento y mantenimiento de las relaciones con sus compañeros/as, empleando para ello una comunicación personal directa: mensajes privados, mensajería instantánea o comunicación de telefonía móvil.

Messing around o "jugar un poco" es el comienzo de una forma más intensa de participación, donde los jóvenes tienen interés en el funcionamiento, medios y contenidos de la tecnología. Esta orientación hacía el interés es apoyada por el "acceso a recursos en línea, recursos de producción de medios de comunicación y un contexto social para el intercambio de conocimientos e intereses" (Ito et al, 2008, p. 20). Este tipo de participación implica por lo tanto, intercambios sociales centrados en los nuevos medios y la tecnología, destacando la importancia en dicha participación de la interacción entre pares. Los responsables de la investigación indican que, cuando se les preguntó a los menores como habían aprendido a usar determinadas herramientas, estos respondieron que "un hermano, un primo o un amigo les mostró cómo hacerlo" (Ito et al, 2008, p.26).

El último de los géneros de participación es geeking out o uso intenso de las TIC. Los autores lo definen como "la capacidad de interactuar con los medios de comunicación y la tecnología de una manera intensa, autónoma y orientada por los intereses" (Ito et al, 2008, p.28), generando en los jóvenes una actitud de compromiso y responsabilidad con la tecnología y con aquellos sujetos participantes en la misma.

Los menores incluidos en esta clasificación tienen un acceso continuo a los medios digitales, siendo la familia y los pares aquellos facilitadores en el acceso a la tecnología, el conocimiento y las conexiones sociales necesarias. Los jóvenes sienten que las redes de amistad tradicionales se les quedan "pequeñas" a la hora de encaminar sus intereses y motivaciones, por lo que buscan diferentes redes de expertos/as que permitan satisfacer sus necesidades educativas. Una vez inmersos en estos espacios, los jóvenes se involucran en una "red social compleja que opera bajo diferentes conjuntos de jerarquía" (Ito et al, 2008, p.30), siendo la competencia digital uno de los dominios más valorados. 
En la red, los menores también pueden convertirse en expertos de determinados ámbitos de conocimiento, influyendo así, en otros/as usuarios/as. La interacción producida entre ambos se basa en un proceso de negociación y consenso, en la que cada sujeto expone sus ideas y opiniones, contraponiendo las del resto de compañeros/as. El estudio indica que los menores se muestran motivados ante dicha interacción, sobre todo a la hora de desarrollar su identidad y reputación digital (reconocimiento colectivo de sus aportaciones).

Se percibe por lo tanto, la consolidación de los pares o iguales como agentes clave en lo que respecta al uso de las TIC. No solamente pueden garantizar el acceso a estas, sino que orientan y guían a sus compañeros/as a lo largo de su interacción. Se conforman por lo tanto, como un componente fundamental en las RPA de los sujetos de educación obligatoria, ayudando y proponiendo mejoras en su proceso de aprendizaje. La influencia de éstos en el desarrollo de la competencia digital es evidente, puesto que en los entornos virtuales aprenden a resolver problemas de manera conjunta, aportan información y debaten sobre la misma, se comunican unos con otros, etc. Se consolida así, un espacio informal con un gran número de posibilidades educativas para todos los agentes intervinientes, en los cuales la colaboración, retroalimentación, confianza y motivación se erigen como pilares fundamentales.

\subsection{Experiencias con RPA y su influencia en la competencia digital}

A continuación, se recogen una serie de experiencias e iniciativas en las que se valorarán la influencia de las redes personales de aprendizaje en la competencia digital de los menores. A la hora de clasificar los ámbitos de dicha competencia se tendrán en cuenta las dimensiones establecidas en el Proyecto DIGCOMP del año 2013. Su elección se debe a que este se ha consolidado como marco de referencia a nivel europeo, permitiendo así, trasladar los resultados obtenidos a otros ámbitos y contextos de investigación:

- Información: identificar, localizar, recuperar, almacenar, organizar y analizar la información digital, evaluando su finalidad y relevancia.

- Comunicación: comunicar en entornos digitales, compartir recursos a través de herramientas en línea, conectar y colaborar con otros a través de herramientas digitales, interactuar y participar en comunidades y redes; conciencia intercultural.

- Creación de Contenidos: crear y editar contenidos nuevos (textos, imágenes, videos...), integrar y reelaborar conocimientos y contenidos previos, realizar producciones artísticas, contenidos multimedia y programación informática, saber aplicar los derechos de propiedad intelectual y las licencias de uso.

- Seguridad: protección personal, protección de datos, protección de la identidad digital, uso de seguridad, uso seguro y sostenible

- Resolución de problemas: identificar necesidades y recursos digitales, tomar decisiones a la hora de elegir la herramienta digital apropiada, acorde a la finalidad o necesidad, resolver problemas conceptuales a través de medios digitales, resolver problemas técnicos, uso creativo de la tecnología, actualizar la competencia propia y la de otros.

Se plasma en primer lugar, la investigación Comunidades Virtuales de Jóvenes: hacer visible sus aprendizajes y saberes (2015) del Grupo Esbrina (Barcelona) con propuestas de estudio centradas en las consecuencias del uso de las TIC dentro y fuera de la escuela. Esta investigación es de especial interés, puesto que indaga acerca de la influencia que tienen las comunidades virtuales -representación del PLN- en el proceso de aprendizaje de los sujetos. 
Además, es de relevancia la propuesta metodológica planteada -de carácter cualitativo-, ya que sitúa a los adolescentes en el plano principal de la investigación.

A pesar de ser un proyecto de reciente creación, se han localizado una serie de resultados parciales, producto de analizar dos comunidades virtuales muy frecuentadas por jóvenes españoles, pertenecientes cada una de ellas a un ámbito de interés distinto. A la hora de valorar estas comunidades, se han tenido en cuenta dimensiones como las prácticas de los/as usuarios/as, las relaciones generadas entre ellos, así como con el conocimiento y los medios de los que disponen.

La primera de las comunidades virtuales analizadas ha sido "Instructables", una plataforma formada por más de dos millones de usuarios/as y centrada en "explorar los proyectos realizados por otros y publicados en la web, así como compartir los proyectos de quienes los crean" (Ribera, Miño y Carrasco, 2016, p.15). Debido al carácter multidisciplinar de esta comunidad, se establecen ocho áreas de trabajo: tecnología, talleres, manualidades, hogar, comida, juegos, exteriores y disfraces. Además, ésta ofrece la posibilidad de formar parte de grupos y participar en foros relativos a los ámbitos explorados en la comunidad.

"Instructables" establece unas reglas de conducta en relación a la actividad de los sujetos en la plataforma y sobre el uso que estos deben hacer de los recursos y medios facilitados. Se destaca por ejemplo la prohibición de "subir" contenidos amenazantes, fraudulentos o que no respeten los derechos de autor. Además, los usuarios no podrán hacerse pasar por otra persona o recolectar información y datos personales sobre los restantes usuarios/as. Se percibe así, un intento por desarrollar la dimensión "seguridad" (Proyecto DIGCOMP) por medio de los requisitos y políticas de privacidad de la plataforma. El aprendizaje de estas normas podrá ser transferido a otros entornos como: redes sociales, foros, blogs, etc. aprendiendo a respetar el trabajo de los demás y responsabilizándose al mismo tiempo, de las propias acciones.

Debido a que esta comunidad se basa en la idea del "do it yourself" (hazlo tú mismo) fabricación o reparación de cosas por uno mismo- se potencia el ámbito de la "creación de contenidos" y "resolución de problemas". De este modo, se elaboran contenidos nuevos que permitan dar respuesta a una serie de problemáticas o situaciones cotidianas; valorando para ello, el formato de creación y los medios digitales que facilitarán su elaboración.

"Dibujando" es la segunda comunidad virtual analizada por el Grupo de Investigación Esbrina. Ésta se conforma como espacio de colaboración entre aquellas personas interesadas por el conocimiento artístico, compartiendo y valorando las distintas producciones visuales.

Sin duda, la dimensión de la "comunicación" es la más presente y reivindicada en esta plataforma, ya que se conforma un espacio abierto y de diálogo donde los menores pueden contribuir y participar de manera activa en la creación de conocimiento. En base a esto, los individuos comparten -mediante los foros y las galerías- todo tipo de recursos: portafolios, infografías, tutoriales de programas de diseño, etc., permitiéndoles ahondar en aspectos poco conocidos o sobre los que quieren ampliar conocimiento.

Puesto que la comunidad se centra en la elaboración de recursos visuales, se fomenta también la dimensión de "creación de contenidos". En este punto, es importante que los sujetos conozcan el diferente software que le permitan realizar con creatividad y calidad diferentes producciones artísticas.

Al igual que en la plataforma anterior se tienen en cuenta aspectos relacionados con la "seguridad": falta de respeto entre usuarios/as, publicación de contenido ilegal, uso indebido del material de otras personas, etc. De esta manera, los menores desarrollan una serie de 
conocimientos, habilidades y actitudes con respecto a la propiedad intelectual de los contenidos, percibiendo la importancia que tienen en este caso, las licencias de uso o la protección personal de los datos.

También la investigación de Herrero y Alcaide-Pulido (2014), aborda la situación de los niños/as y jóvenes como actores y creadores de contenidos en Internet. Para ello, analizan la experiencia realizada por medio de la plataforma "Cibercorresponsales", destinada a que sujetos menores de 18 años compartan sus experiencias, vivencias, opiniones, etc. sobre aquellos aspectos que "despierten" su interés y motivación. Esta plataforma -con un funcionamiento similar al de una red social- permite que cada sujeto elabore un blog sobre cualquier temática, pudiendo al mismo tiempo, leer y comentar las producciones del resto de sus compañeros/as.

Estas autoras han procedido a valorar los comentarios enviados y recibidos entre sujetos, determinando así, la influencia real de esta red de aprendizaje en los jóvenes. Para su análisis y posterior clasificación se han establecido -mediante un proceso inductivo- tres variables muy presentes en la participación de los sujetos:

- Motivación: se busca determinar el nivel de apoyo y colaboración existente entre los menores. Se percibe esta categoría en comentarios como:

"¡Qué buena información Pablo! Tus artículos son cada vez más complejos, te los curras y se nota. Además, son muy interesantes. jSigue así!".

- Creación: se analiza la información generada por los jóvenes en Internet, valorando los diferentes formatos en función de la plataforma y la tecnología utilizada para la construcción de mensajes: podcast, wikis, videoblogs, etc. Dentro de esta temática, se recoge el siguiente comentario:

"El artículo está muy bien, pero hay páginas mejores que la Wikipedia. Te paso más información el fin de semana y la cambias, si quieres... Disfruta del sábado".

- Aprendizaje: categoría referida a la construcción colectiva de conceptos, valores, actitudes, etc. ante un determinado tema. Algunos mensajes representativos de la presente clasificación son:

"Me gusta la manera en la que describes el atletismo, porque yo soy una de esas personas que lo miraba de esa manera, y ahora empiezo a entender un poco mejor como es el atletismo. Gracias, y por cierto, molan mucho las fotos". :)

Los anteriores comentarios recogidos a modo de ejemplo, aportan luz acerca de las competencias desarrolladas por los menores. Detrás de cada aportación o mensaje, los sujetos realizan una serie de procesos educativos previos que les permiten comunicarse con éxito con el resto de compañeros/as. Se perciben de este modo, competencias relacionadas con "la lectura, la capacidad de preguntar, analizar, criticar y mejorar la capacidad expresiva y las habilidades comunicativas para contar los resultados de su trabajo" (Herrero y Alcaide-Pulido, 2014, p.6).

Se percibe una gran influencia de esta red personal de aprendizaje en el desarrollo de los ámbitos de "información", "comunicación" y "creación de contenidos" establecidos por el Proyecto DIGCOMP. De este modo, los menores buscan y seleccionan aquella información que sea de su interés, valorando también el impacto que ésta puede tener en el resto de compañeros/as (información). Esta experiencia se fundamenta en el ámbito de la "comunicación", puesto que la conexión y colaboración entre sujetos es fundamental para garantizar la construcción de conocimiento. Se produce también, un desarrollo en la "creación 
de contenidos", ya que los sujetos integran y reelaboran la información seleccionada, con el objetivo de crear contenidos nuevos o reeditar los ya existentes.

Resultados similares se recogen en la tesis de Gil Mediavilla (2012): "Desarrollo de Entornos Personales de Aprendizaje (PLEs) para la mejora de la competencia digital. Estudio de caso en una escuela media italiana". Este documento plasma la experiencia realizada con niños/as de entre 11 y 14 años, basada en la consolidación de los PLE como espacios de aprendizaje continuo. La gran presencia de herramientas 2.0. ha permitido disponer de un gran número de posibilidades a la hora de comunicarse, crear contenidos, etc. El autor de esta tesis, destaca el impacto de los entornos y redes personales de aprendizaje en el desarrollo del ámbito denominado "seguridad" (Proyecto DIGCOMP).

En las diferentes iniciativas que dan cuenta de la influencia de las RPA en los distintos ámbitos de la competencia digital, se observa que el ámbito de la "comunicación" está presente en todas las experiencias, percibiéndolo como un elemento inherente en estas redes. También se le concede relevancia a todo aquello relacionado con la "seguridad" de los menores y sus aportaciones, pudiendo encontrar una posible explicación en el creciente interés de la comunidad educativa en la protección digital del colectivo infantil y juvenil.

\section{DISCUSIÓN Y CONCLUSIONES}

A lo largo de este artículo se ha intentado resaltar el valor de la Red Personal de Aprendizaje como un nuevo contexto educativo caracterizado por la participación y colaboración de todo tipo de personas, así como por su influencia en el aprendizaje informal de los sujetos (Coffield, 2000).

La participación y colaboración de los menores en los diversos espacios ha quedado patente, puesto que estos muestran intención por expandir los entornos sociales y educativos más allá del contexto formal, evitando así, su rigidez y carácter estático. Los espacios virtuales aportan una mayor flexibilidad y adaptabilidad a las características de los sujetos (horarios de acceso, disponibilidad de recursos, competencias previas, etc.), conformando según Castañeda y Adell (2013), su identidad digital en Internet.

Con respecto a las Redes Personales de Aprendizaje de niños/as de entre 12 y 16 años, la mayor parte de los archivos revisados (Domingo, 2008; Ito et al, 2008) conceden importancia a los iguales o pares en el desarrollo de la competencia digital. Debido a que los menores se encuentran en una situación similar (edad, vivencias, intereses, etc.), estos forman parte crucial del proceso de aprendizaje. En Internet, los jóvenes se conforman como guías o expertos/as en un determinado ámbito- el estatus se determina por sus habilidades (Freire, 2009)-, ayudando a los demás a superar los obstáculos o problemas encontrados.

Se ha observado también que, aunque existen ciertas experiencias relativas a la red personal de aprendizaje y los jóvenes, éstas no profundizan en las potencialidades y limitaciones de la misma en el proceso educativo. Los documentos recogidos hasta el momento tienen un carácter predominantemente descriptivo -narración de la experiencia- no contemplando la valoración crítica y reflexiva de la implicación de las redes de aprendizaje en el desarrollo de la competencia digital de los sujetos.

El análisis y valoración de las diferentes experiencias entre iguales o colaborativas ha permitido determinar la gran influencia que tienen en el desarrollo de la competencia digital (Herrero y Alcaide-Pulido, 2014). Se ha partido desde una visión integradora de esta competencia, considerando cada uno de sus componentes como elementos interrelacionados y dependientes los unos de los otros. Se percibe un interés en estas experiencias por garantizar 
el ámbito de la "seguridad" (según el Proyecto DIGCOMP). Una posible explicación de este interés se puede deber al incremento en los últimos años de los riesgos en Internet (ciberacoso, robo de información, grooming, etc.), lo que ha provocado que la mayor parte de la comunidad educativa indique la importancia de garantizar un acceso seguro de los menores a la tecnología.

A pesar de que la "seguridad" ha sido muy destacada en las experiencias recompiladas, los restantes ámbitos también están muy presentes. Como se observa en Ribera, Miño y Carrasco (2016) el desarrollo de una determinada dimensión, lleva implícito la potenciación de las restantes. De este modo, cuando un sujeto quiere publicar un mensaje en un foro ("comunicación"), es preciso que este realice una búsqueda de contenido previa ("información"), para a continuación, elaborar un producto adaptado a sus receptores ("creación de contenido").

A raíz de la valoración de estas iniciativas, se ha percibido una limitación en cuanto a su análisis como entornos de aprendizaje. Consideramos que las redes personales de aprendizaje son espacios inabarcables, conformados en muchas ocasiones, por cientos de interacciones con otras personas. Así, para determinar la influencia real de las mismas, es preciso analizar uno a uno estos comentarios, aportaciones, interacciones, etc., ya que cada uno de ellos puede potenciar ámbitos distintos de la competencia digital.

En definitiva, el presente artículo ha intentado dar respuesta a los interrogantes encuadrados en un ámbito de conocimiento poco desarrollado, con el objetivo de ayudar en la apertura de líneas de investigación actuales y con gran impacto en la comunidad educativa.

\section{REFERENCIAS BIBLIOGRÁFICAS}

Apodaca, P. (2006). Estudio y Trabajo en Grupo. En M. Miguel. (Ed.), Metodologías de enseñanza y aprendizaje para el desarrollo de competencias (pp. 169-190). Madrid: Alianza.

Cabero, J., Barroso, J. y Llorente, M.C. (2010). El diseño de Entornos Personales de Aprendizaje y la formación de profesores en TIC. Digital Education Review, 18, 26-37. Recuperado de: http://greav.ub.edu/der

Camacho, K. (2005). La brecha digital. En A. Ambrosi, V. Peugeot y D. Pimienta. (Eds.), Palabras en juego: enfoques multiculturales sobre las sociedades de la información (pp. 6171). París: CyF Editions.

Castañeda, L. y Adell, J. (2013). Entornos Personales de Aprendizaje: claves para el ecosistema educativo en red. Alcoy: Marfil.

Casquero, O. (2013). Composición y estructura de redes personales en entornos de aprendizaje personales. En L. Castañeda y J. Adell (Eds.), Entornos Personales de Aprendizaje: Claves para el ecosistema educativo en red (pp. 151-160). Alcoy: Marfil

Coffield, F. (2000). The Necessity of Informal Learning. Bristol: The Policy Press.

Couros, A. (2010). Developing personal learning networks for open and social learning. En G. Veletsianos. (Ed.), Emerging technologies in distance education (pp. 109-128). Canada: AU Press.

Cross, J. (2006). Informal Learning: Rediscovering the Natural Pathways That Inspire Innovation and Performance. San Francisco: Pfeiffer. 
Cuadros Muñoz, R. (2015). Aprendizaje informal y construcción de PLN vía Twitter. Un estudio de caso. Edutec. Revista Electrónica de Tecnología Educativa, 51, 1-18. Doi: 10.21556/edutec.2015.51.53

Cuseo, J.B. (1996). Cooperative Learning: A Pedagogy for Addressing Contemporary Challenges and Critical Issues in Higher Education. Marymount College: New Forums Press.

Domingo, J. (2008). El aprendizaje cooperativo. Cuadernos de Trabajo Social, 21, 231246. Recuperado de: http://revistas.ucm.es/index.php/CUTS/article/view/8377

Fernández Núñez, L. (2006). ¿Cómo analizar los datos cualitativos? Butlletí LaRecerca, 6 , 1-13. Recuperado de: http://www.ub.edu/ice/recerca/fitxes/fitxa7-cast.htm

Ferrari, A. (2013). DIGCOMP: A Framework for Developing and Understanding Digital Competence in Europe. Sevilla: Joint Research Centre.

Freire, J. (2009). Cultura digital y prácticas creativas en educación. Revista de Universidad y Sociedad del Conocimiento, 6(1), 1-52. Recuperado de: http://rusc.uoc.edu/index.php/rusc/article/viewFile/v6n1-freire/v6n1-cultura-digital-practicascreativas-en-educacion

Galvan, J. L. (2006). Writing literature reviews: A guide for students of social and behavioural sciences. Los Ángeles: Pyrczak Publishing.

Gil Mediavilla, M. (2012). Desarrollo de Entornos Personales de Aprendizaje (PLEs) para la mejora de la competencia digital. Estudio de caso en una escuela media italiana [Tesis doctoral]. Burgos: Universidad de Burgos.

Green, B. N., Johnson, C. D., y Adams, A. (2006). Writing narrative literature reviews for peer-reviewed journals: secrets of the trade. Journal of Chiropractic Medicine, 5(3), 101-117. Doi: 10.1016/S0899-3467(07)60142-6.

Herrero, P. y Alcaide-Pulido, P. (2014). La comunicación digital en el aprendizaje entre iguales. Comunicación presentada en La sociedad ruido: entre el dato y el grito, Santiago de Compostela (España). Resumen recuperado de: http://www.revistalatinacs.org/068/cuadernos/cac53.pdf

Ito, M., Horst, H., Bittanti, M., Boyd, D., Herr-Stephenson, B.,Lange, P.G., ... y Tripp, L. (2008). Living and Learning with New Media: Summary of Findings from the Digital Youth Project. Chicago: The MacArthur Foundation.

Melnyk, B. (2005). Evidence-Based Practice in Nursing and Healthcare. Philadelphia: Lippincott Williams y Wilkins.

OCDE (2014). Education at a glance 2014. OECD Indicators: OECD Publishing.

Rajagopal, K., Joosten-ten Brinke, D., Van Bruggen, J., y Sloep, P. B. (2011). Understanding personal learning networks: Their structure, content and the networking skills needed to optimally use them. First Monday, 17(1), 1-12. Doi: 10.5210/fm.v17i1.3559

Ribera, P., Miño, R. y Carrasco, S. (2016). Las comunidades virtuales como posibles espacios de visibilización y participación social para los jóvenes. Comunicación presentada en XII Congreso Español de Sociología. Grandes transformaciones sociales, nuevos desafíos para la sociología, Gijón (España). Resumen recuperado de: http://fes- 
sociologia.com/comunidades-virtuales-posibles-espacios-de-visibilizacion-y-particip/congresspapers/3458/

Richardson, W. y Mancabelli, R. (2011). Personal learning networks. Bloomington: Solution Tree Press.

Ruiz Olabuénaga, J. I. (1999). Metodología de la investigación cualitativa. Bilbao: Universidad de Deusto.

Salinas, J. (2004). Innovación docente y uso de las TIC en enseñanza universitaria. Revista de Universidad y Sociedad del Conocimiento, 1(1), 1-16. Recuperado de: http://www.uoc.edu/rusc/dt/esp/salinas1004.html

Sangrà, A. y Wheeler, S. (2013). New Informal Ways of Learning: Or Are We Formalising the Informal? Revista de Universidad y Sociedad del Conocimiento, 10(1), 107-115. Doi: 10.7238/rusc.v10i1.1689.

\title{
INFORMACIÓN SOBRE LOS AUTORES
}

\section{Sandra Dorado Gómez}

Universidad de Santiago de Compostela

\begin{abstract}
Sandra Dorado Gómez es graduada en Pedagogía (2015) en la Facultad de Ciencias de la Educación de la Universidad de Santiago de Compostela. Completó su formación realizando el Máster en Procesos de Formación (RD 1393/2007), finalizando esta etapa con un Trabajo Fin de Máster sobre la influencia de las Redes Personales de Aprendizaje (Personal Learning Network) en la competencia digital del alumnado de educación obligatoria.

Tras unos meses participando en el grupo de investigación con una Beca de Colaboración (2014-2015), actualmente se encuentra contratada por el Ministerio de Educación, Cultura y Deporte a través de su programa de Formación de Profesorado Universitario (FPU 2015) por la que se encuentra desarrollando su actividad investigadora dentro del grupo y realizando su tesis doctoral centrada en el estudio de la "Competencia digital y redes personales de aprendizaje de estudiantes en educación obligatoria".
\end{abstract}

\section{Adriana Gewerc Barujel \\ Universidad de Santiago de Compostela}

Dra. Adriana Gewerc Barujel nació en Córdoba, Argentina. Es licenciada en Ciencias de la Educación por la Universidad Nacional de Córdoba- Argentina (1982) y Doctora en Pedagogía (1998) por la Universidad de Santiago de Compostela. Forma parte del grupo de investigación Stellae. Actualmente trabaja como profesora titular en esta universidad impartiendo las asignaturas Tecnología Educativa y Multimedia y Software Educativo. Sus líneas de investigación se centran en la problemática y el significado de la integración de las tecnologías en las organizaciones educativas y sus implicaciones en la enseñanza. En ese contexto en el último tiempo explora para la docencia y la investigación, la utilización de nuevos entornos de enseñanza y aprendizaje. Ha dirigido tesis doctorales sobre estas temáticas y tiene libros y artículos publicados que evidencian el trabajo realizado en estos años.

Web: http://webspersoais.usc.es/persoais/adriana.gewerc/

\section{(cc) BY-NC}

Los textos publicados en esta revista están sujetos a una licencia de Reconocimiento 4.0 España de Creative Commons. Puede copiarlos, distribuirlos, comunicarlos públicamente y hacer obras derivadas siempre que reconozca los créditos de las obras (autoría, nombre de la revista, institución editora) de la manera especificada por los autores o por la revista. La licencia completa se puede consultar en: Licencia Creative Commons Atribución-NoComercialCompartir por igual 4.0 Internacional. 